\title{
Desempeño de discos segmentados de diamante en el corte de hormigón
}

\section{Performance of segmented diamond discs in concrete cutting}

\author{
Vitaliy Martynenko ${ }^{1}$, Nazareno Antúnez ${ }^{1}$, Alejandra Benítez ${ }^{2}$, \\ Daniela Perez ${ }^{1}$,Daniel Martínez Krahmer ${ }^{1}$, Gustavo Genovese ${ }^{3}$
}

\footnotetext{
${ }^{1}$ Centro de Investigación y Desarrollo en Mecánica, Instituto Nacional de Tecnología Industrial, Avenida General Paz 5445, Miguelete, Buenos Aires, Argentina.

e-mail: vmart@inti.gob.ar; nantunez@inti.gob.ar; danielap@inti.gob.ar; mkrahmer@inti.gob.ar

${ }^{2}$ Centro de Investigación y Desarrollo en Construcciones, Instituto Nacional de Tecnología Industrial, Avenida General Paz 5445, Miguelete, Buenos Aires, Argentina.

${ }^{3}$ All Import SA, Paraná 4532, 1605, Munro, Buenos Aires, Argentina. e-mail: gustavo@e-bremen.com
}

\section{RESUMEN}

Los discos de diamante segmentados se emplean en el corte de materiales abrasivos y frágiles, como son la mampostería, el hormigón y los mármoles. Su constante evolución, durante los últimos 15 años, ha permitido incrementar la efectividad de estas operaciones. Sin embargo, y a pesar de su difusión, todavía no existe un método normalizado que evalúe su rendimiento en condiciones representativas de un servicio normal.

En este trabajo, se presenta un procedimiento completo sobre como evaluar el desempeño de estos discos. Para tal fin fue necesario desarrollar: una máquina de corte por Control Numérico Computarizado (CNC), un material de ensayo, las condiciones operativas, el criterio de interrupción, y las formas de evaluación (desgaste y profundidad de corte).

Se ensayaron cuatro marcas de discos segmentados de diamante de $115 \mathrm{~mm}$ de diámetro, designados como A, B, C y D respectivamente, con tres réplicas por cada una de ellas. Las pruebas se realizaron cortando un total de 45 bloques de hormigón prismáticos de 290 x 260 x $75 \mathrm{~mm}$.

En cuanto al desgaste, mientras los discos de los grupos A y D mostraron un comportamiento similar, el grupo B resultó el de menor performance, y el C alcanzó la mejor.

En relación a la capacidad de cortar mayores profundidades, los discos de los grupos A y B fueron aquellos de mejor rendimiento.

Si el comportamiento de los discos se juzga por la relación profundidad de corte / desgaste, el mejor comportamiento global lo tuvo el grupo C.

De acuerdo con los resultados obtenidos, es posible afirmar que, los métodos de variación del diámetro y de pérdida de peso, pueden ser considerados equivalentes.

Para finalizar, la observación de las superficies de corte de este tipo de discos, empleando microscopía electrónica de barrido, ha permitido justificar la capacidad cortante observada durante los ensayos.

Palabras clave: discos, diamantados, corte, hormigón.

\section{ABSTRACT}

Segmented diamond discs are used in cutting abrasive and fragile materials, such as masonry, concrete and marble. Their constant evolution over the past 15 years has allowed increasing the effectiveness of these operations. However, despite being widely used, there is still no standard test to assess their performance in representative conditions of a normal service.

In this work a complete procedure to evaluate the disc performance is presented. To that end it was necessary to develop: a Computer Numeric Control (CNC) cutting machine, a test material, the operating conditions, the stopping criterion, and the evaluation methods (wear and depth of cut).

Four brands of segmented diamond discs of $115 \mathrm{~mm}$ of diameter, nominated A, B, C and D respectively, 
were tested three times. . The tests were made cutting 45 prismatic blocks of concrete, $290 \times 260 \times 75 \mathrm{~mm}$ in size.

As for the wear, the discs in groups A and D showed similar performance, while group B scored last, and group $\mathrm{C}$ scored best.

As regards to the aptitude to cut major depths, groups A and B showed better performance.

When the behavior of the discs was evaluated by the depth of cut to wear ratio, the best performance was achieved with group $\mathrm{C}$.

In accordance with the obtained results, it is possible to affirm that, the methods of diametral change and weight loss used to quantify the wear of the discs, can be considered equivalent.

Finally, the observation of the cut surfaces of this type of discs, using Scanning Electron Microscopy, allows justifying the cutting capability observed during the tests.

Keywords: discs, diamond, cutting, concrete.

\section{INTRODUCCIÓN}

Los discos de corte, representan una de las herramientas habitualmente empleadas en el corte de materiales abrasivos y frágiles, como pueden ser la mampostería, el hormigón y los mármoles. Inclusive, durante los últimos 15 años, los discos con segmentos diamantados (que consisten en partículas de diamante embebidas en una matriz metálica), han permitido incrementar sustancialmente la efectividad de estas operaciones [1]. Sin embargo, y a pesar de su amplia difusión, todavía no existe un ensayo normalizado que evalúe el rendimiento de estos discos, en condiciones representativas a las de un servicio normal.

¿Hacia dónde apuntan las investigaciones publicadas hasta el momento? A grandes rasgos, pueden ser agrupadas en dos sentidos: a) establecer los medios técnicos para extender la durabilidad mediante la realización de ensayos de desgaste [1-4] y b) acentuar su efectividad analizada en términos del consumo de energía [2-7]; fuerzas de corte [1,3,-7]; vibraciones [5,6]; e influencia de los fluidos de corte [3].

En estricta relación a los ensayos de desgaste, los investigadores se centraron en probar: a) distintos aglomerantes (variantes en peso de compuestos de cobalto, cobre, hierro, carburo de tungsteno, etc); b) partículas de diamante de tamaños variados; c) diferentes materiales (diversos tipos mármol y vidrio); d) condiciones de corte (velocidad tangencial, velocidad de avance, profundidad de pasada) y e) lubricación.

Una de las modalidades relativamente sencillas para obtener un valor de desgaste resulta de medir como varia el peso del disco con el uso [1,2]

En tanto que, una forma sofisticada que contempla la variación del diámetro del disco, pero que sólo puede ser aplicada en un laboratorio, es efectuando las determinaciones con un sistema de medición láser $[3,4]$.

En consecuencia, en este trabajo hemos desarrollado una metodología de ensayo propia, que permite evaluar y comparar el desempeño de discos de distintos fabricantes, y cuyo criterio de interrupción se fijó en base a la superficie de hormigón cortada. Asimismo, y con el objeto de aportar simplicidad a las mediciones de desgaste, se propone establecerlas también por la variación del diámetro exterior.

Por último también se contribuye con una manera sobre como cuantificar cuando un disco posee una capacidad cortante superior, en términos de la profundidad máxima que permite alcanzar, sin poner en riesgo la vida útil de la máquina amoladora.

\section{MATERIALES Y MÉTODOS}

Dada la inexistencia de una norma para realizar un ensayo de desempeño de este tipo de discos, , fue necesario en primer lugar diseñar un mortero para la fabricación de las probetas a ser ensayadas. Luego se dosificó una mezcla de hormigón con piedra partida, a partir del cual, se elaboraron 45 bloques prismáticos, cuyas dimensiones aproximadas resultaron ser de $290 \times 260 \times 75 \mathrm{~mm}$.

El diseño de este material a ser cortado, fue realizado por especialistas del laboratorio de Tecnología del Hormigón del Centro de Construcciones del Instituto Nacional de Tecnología Industrial.

La dosificación empleada fue la siguiente: 
Tabla 1: Dosificación usada para las probetas de hormigón

\begin{tabular}{l|l}
\hline MATERIAL & Pss $\left[\mathbf{k g} / \mathbf{m}^{\mathbf{3}}\right]$ \\
\hline Agua & 178 \\
\hline Cemento CPF 40 & 312 \\
\hline Arena silícea de río & 645 \\
\hline Piedra Partida 6-20 & 1239 \\
\hline Relación agua: cemento & 0,57 \\
\hline Aditivo Superfluidificante (dosis 1\% en masa de cemento) & 3,1 \\
\hline Sumatoria Peso de materiales & 2377 \\
\hline
\end{tabular}

Referencias: Psss = peso saturado a superficie seca

Los bloques prismáticos fueron desmoldados con fecha 26/05/15 y se sometieron a curado por inmersión en agua saturada con cal a $(23 \pm 2){ }^{\circ} \mathrm{C}$ hasta la fecha 24/06/15.

Con el objeto de caracterizar el comportamiento del hormigón, se moldearon 8 (ocho) probetas cilíndricas de $\phi 150$ × $300 \mathrm{~mm}$, que se utilizaron para determinar la resistencia a compresión, dos por cada edad de ensayo, a los 5, 14, 21 y 28 días.

En la tabla 2 se incluyen los resultados obtenidos. Las resistencias medias se calcularon como el promedio de los valores individuales:

Tabla 2: Valores obtenidos para la resistencia a la compresión del hormigón

\begin{tabular}{l|l|l|l|l|l|l|l}
\hline \multirow{2}{*}{ IDENTIFICACIÓN } & $\begin{array}{l}\text { FECHA DE } \\
\text { MOLDEO }\end{array}$ & $\begin{array}{l}\text { FECHA DE } \\
\text { ROTURA }\end{array}$ & $\begin{array}{l}\mathbf{D}_{\mathbf{M}} \\
{[\mathbf{m m}]}\end{array}$ & $\begin{array}{l}\mathbf{P} \\
{[\mathbf{k N}]}\end{array}$ & $\begin{array}{l}\text { EDAD } \\
\text { [días] }\end{array}$ & $\begin{array}{l}\sigma_{\mathbf{c}} \\
\text { [MPa] }\end{array}$ & $\begin{array}{l}\sigma_{\text {cmed }} \\
\text { [MPa] }\end{array}$ \\
\hline 1 & $21 / 05 / 15$ & $26 / 05 / 15$ & 150,1 & 305,3 & 5 & 17,3 & \multirow{2}{*}{17,6} \\
\hline 2 & $21 / 05 / 15$ & $26 / 05 / 15$ & 149,6 & 313,7 & 5 & 17,8 & \\
\hline 3 & $21 / 05 / 15$ & $04 / 06 / 15$ & 149,8 & 422,5 & 14 & 24,0 & \multirow{2}{*}{24,1} \\
\hline 4 & $21 / 05 / 15$ & $04 / 06 / 15$ & 150,8 & 432,4 & 14 & 24,2 & \\
\hline 5 & $21 / 05 / 15$ & $11 / 06 / 15$ & 150,0 & 441,0 & 21 & 25,0 & \multirow{2}{*}{25,4} \\
\hline 6 & $21 / 05 / 15$ & $11 / 06 / 15$ & 150,5 & 457,6 & 21 & 25,7 & \\
\hline 8 & $21 / 05 / 15$ & $18 / 06 / 15$ & 149,6 & 450,8 & 28 & 25,6 & \multirow{2}{*}{26,7} \\
\hline 8 & $21 / 05 / 15$ & $18 / 06 / 15$ & 149,7 & 488,0 & 28 & 27,7 & \\
\hline
\end{tabular}

Sobre cada una de las probetas prismáticas, se efectuaron cortes longitudinales en las dos caras opuestas de mayor área, a razón de 28 cortes lineales, separados unos 9 mm entre sí.

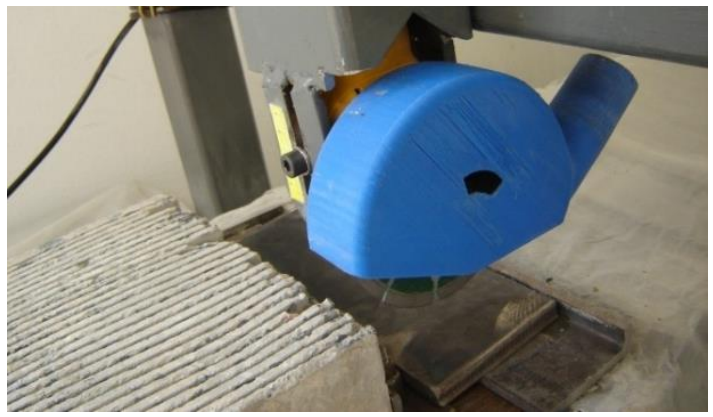

Figura 1: Bloque de hormigón con cara superior ranurada.

Para poder evaluar la capacidad de corte de los discos, se adaptó una máquina existente, sobre la base de una mesa en cruz CNC en desuso, provista de servomotores de corriente continua, a la cual se incorporó un control con hardware del tipo Arduino.

Sobre este "banco de ensayo", se implementó un puente fijo de tubos rectangulares, vinculado a la estructura de la máquina, con un dispositivo sostén para una amoladora angular manual marca Dewalt para discos de $115 \mathrm{~mm}$ de diámetro, $900 \mathrm{~W}$ de potencia y $11000 \mathrm{rpm}$ de velocidad de rotación (este dispositivo permite regular la altura del disco, para definir la profundidad de corte h sobre la probeta). 
El material a cortar fue fijado con una morsa manual, sobre la mesa del banco.

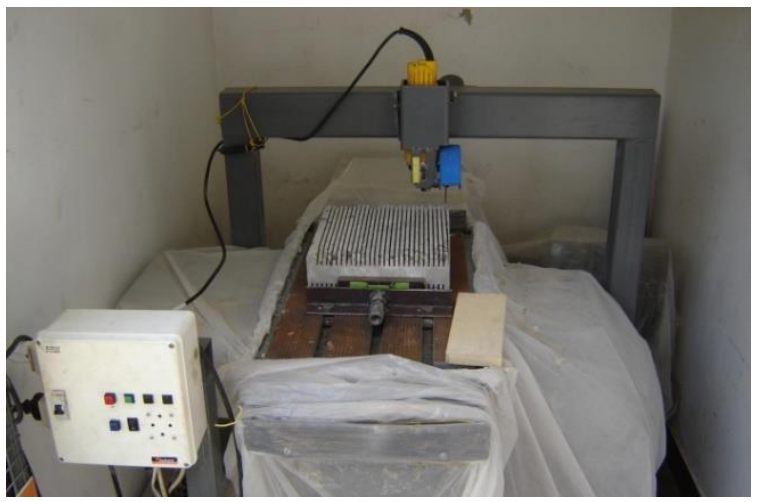

Figura 2: Banco de ensayo de discos de diamante segmentados.

El control fue programado para proveer a la mesa de movimientos en zigzag (movimientos lineales longitudinales en dos sentidos con un desplazamiento transversal entre cada pasada longitudinal, para aprovechar mejor la superficie del bloque).

Antes de efectuar los ensayos, se realizaron pruebas exploratorias, con la finalidad de definir la velocidad de avance máxima a emplear durante los ensayos, manteniendo una profundidad de corte h en $20 \mathrm{~mm}$ (con disco nuevo), y no superando un consumo de corriente de 4 A en la amoladora. Resultó así una velocidad de avance de $390 \mathrm{~mm} / \mathrm{min}$.

Por último, y con el objeto de darle una mayor amplitud a los análisis efectuados, se ensayaron 4 marcas de discos segmentados de diamante de $115 \mathrm{~mm}$ de diámetro, de las que comúnmente se encuentran en el mercado argentino, siendo designadas como A, B, C y D respectivamente, con tres réplicas por cada una.

Para poder determinar el desgaste de los discos se realizó la determinación de su peso, con una balanza Moretti OAC-2.4 de 0,2 g de apreciación y la medición del diámetro exterior con un calibre digital NSK de 0,01 mm de apreciación (fueron efectuadas antes y después de los ensayos).

Nota 1: En razón que la altura de los bloques de hormigón no resultó constante (en todo el lote varió entre un mínimo de $85 \mathrm{~mm}$ y un máximo de $90 \mathrm{~mm}$ ), fue necesario establecer como criterio de detención de ensayo una cantidad de superficie lateral cortada (se obtiene de multiplicar la cantidad de cortes, por la longitud 1 de cada corte, por la profundidad h), resultando de aproximadamente de unos $650000 \mathrm{~mm}^{2}$.

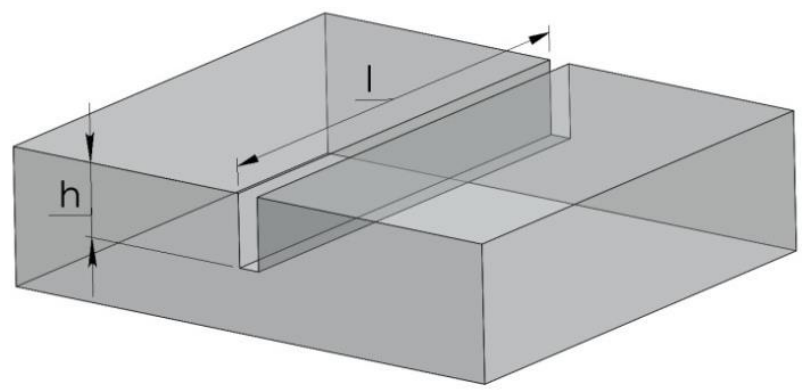

Figura 3: Esquema del bloque con la superficie lateral.

Nota 2: Otro aspecto importante a considerar fue que, si bien en todos los casos, se empezó a cortar con una profundidad h de $20 \mathrm{~mm}$ (disco nuevo), después de realizar varios cortes, algunos discos perdieron su capacidad inicial (se percibió a través de un aumento del consumo de corriente o una detención del giro del disco y/o del avance de la mesa). En consecuencia, para poder avanzar con los ensayos fue necesario bajar la profundidad de corte h, en algunos casos hasta $8 \mathrm{~mm}$. Entonces, esta situación reforzó la necesidad de establecer un criterio de interrupción por superficie lateral cortada. 
A los efectos de establecer el motivo de la diferencia de capacidad de corte de las distintas marcas en términos de la profundidad de pasada h, se obtuvieron imágenes por microscopía electrónica de barrido. El microscopio utilizado fué un SEM 505 de marca Philips. La tensión del emisor fué de $25 \mathrm{kV}$. La medición se realizó mediante el software EDAX Genesis.

\section{RESULTADOS}

En la tabla 3 se presentan los valores de profundidad de corte para cada disco ensayado (corriente $<4 \mathrm{~A}$ ).

Entonces, para poder comparar la degradación de los discos se tuvo que mantener constante la superficie lateral cortada en unos $650000 \mathrm{~mm}^{2}$, equivalente a una longitud cortada de $32500 \mathrm{~mm}$ para una profundidad de pasada $\mathrm{h}$ de $20 \mathrm{~mm}$.

En razón de lo expuesto, hubo discos que cortaron dos bloques (los que mantuvieron durante todo el proceso una profundidad de $20 \mathrm{~mm}$ ), y otros cinco bloques (aquellos que solo pudieron cortar unos $8 \mathrm{~mm}$ ).

Tabla 3: Profundidad de pasada máxima por disco

\begin{tabular}{l|l|l}
\hline DISCO & PROFUNDIDAD $\mathbf{h}(\mathbf{m m})$ & PROFUNDIDAD PROMEDIO $\mathbf{h}(\mathbf{m m})$ \\
\hline $\mathrm{A}_{1}-\mathrm{A}_{2}-\mathrm{A}_{3}$ & $20-17-18$ & 18,3 \\
\hline $\mathrm{B}_{1}-\mathrm{B}_{2}-\mathrm{B}_{3}$ & $20-20-20$ & 20,0 \\
\hline $\mathrm{C}_{1}-\mathrm{C}_{2}-\mathrm{C}_{3}$ & $16-15-11$ & 14,0 \\
\hline $\mathrm{D}_{1}-\mathrm{D}_{2}-\mathrm{D}_{3}$ & $9-8-8$ & 8,3 \\
\hline
\end{tabular}

Los resultados de desgase diamtral se presentan en forma grafica en la figura 4 :

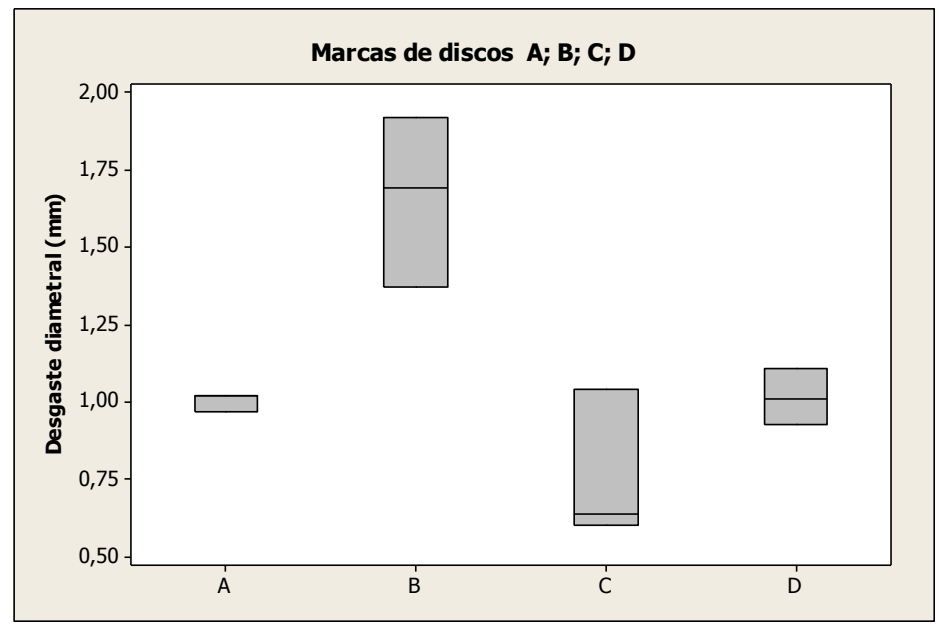

Figura 4: Diagrama de cajas del "Desgaste diametral".

De la observación del diagrama de cajas del desgaste diametral se desprende que, las marcas A, C y D, no pudieron diferenciarse entre sí. La única que presentó diferencias fue la B, que fue a su vez aquella con mayor tasa de desgaste. La marca con menor dispersión resultó ser la A, mientras que la de mayor fue la B.

Para

A continuación se presentan los resultados de pérdida de peso en forma gráfica: 


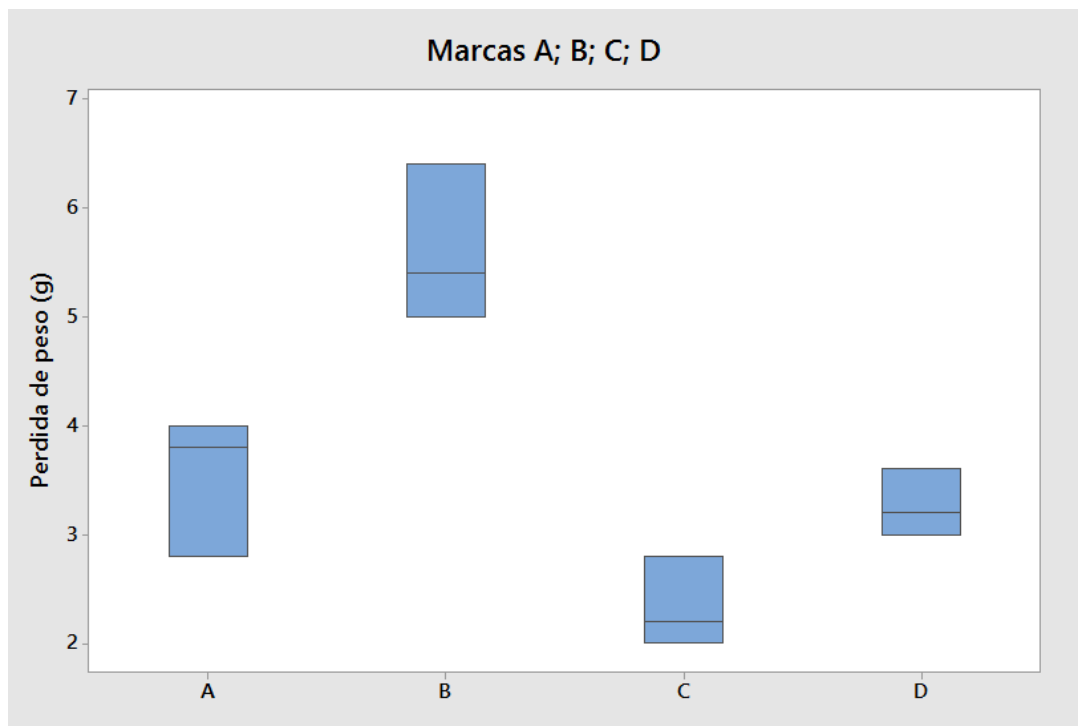

Figura 5: Diagrama de cajas de "Pérdida de peso".

De la observación del diagrama de cajas de pérdida de peso se desprende que, las marcas A y D, no pueden diferenciarse entre sí. Que las B y C presentaron diferencias siendo esta ultima la que mostró el menor valor de desgaste, mientras que la $\mathrm{B}$, fue aquella de mayor tasa. La marca con menor dispersión resultó ser la $\mathrm{D}$, en tanto que, la de mayor fue la $\mathrm{B}$.

\section{DISCUSIÓN}

Es evidente que los gráficos correspondientes al desgaste diametral y por pérdida de peso muestran un comportamiento de similar tendencia. Entonces, ya que es más común disponer de un calibre que de una balanza de precisión, se efectuó un diagrama de "Variación diametral" vs "Pérdida de peso", para establecer si ambos métodos pueden ser utilizados de forma equivalente. Asimismo, se realizó un análisis de regresión lineal, mediante el cual se obtuvo la recta de regresión y el $\mathrm{R}^{2}$, para justificar esta posible equivalencia.

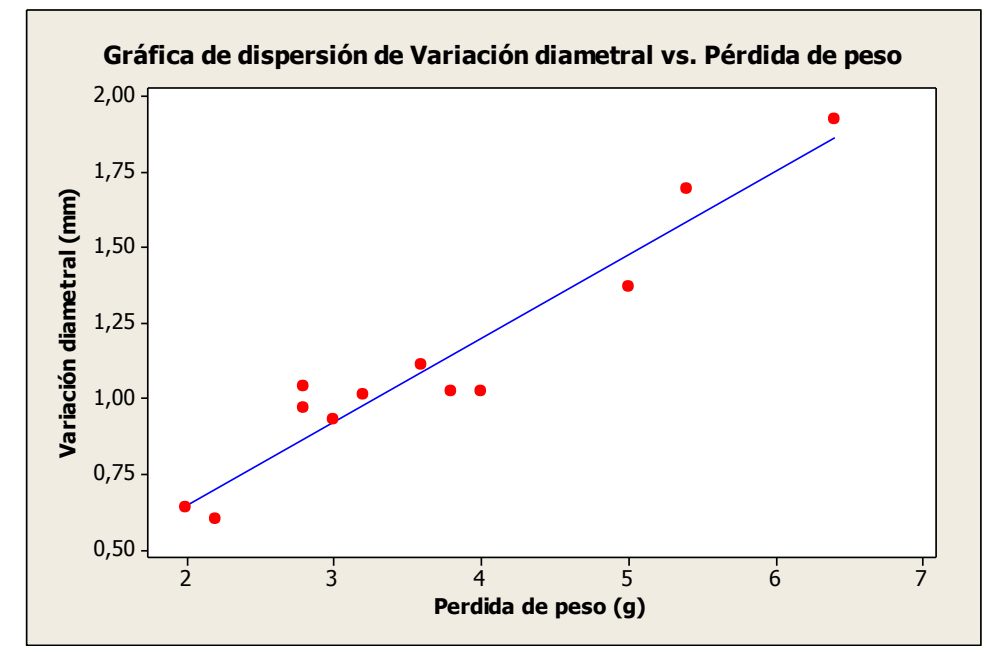

Figura 6: Diagrama de "Variación diametral" vs "Pérdida de peso" para los discos evaluados.

Del estudio estadístico surgen la ecuación de la recta de regresión y su $\mathrm{R}^{2}=92,3 \%$.

$V=0,09+0,28 P$ 
Donde:

$V=$ variación diametral.

$P=$ pérdida de peso.

Resulta evidente que, existe una buena correlación entre los dos métodos utilizados para cuantificar el desgaste de los discos, y en consecuencia, la evaluación por pérdida de peso, puede ser reemplazada por una que considere la variación diametral.

Por otra parte, ya que un usuario de estas herramientas buscaría cortar la mayor profundidad posible mientras el disco se desgaste poco, mostramos a continuación un gráfico de Profundidad / desgaste, el primero de ellos referido a la variación diametral, y el segundo, a la pérdida de peso.

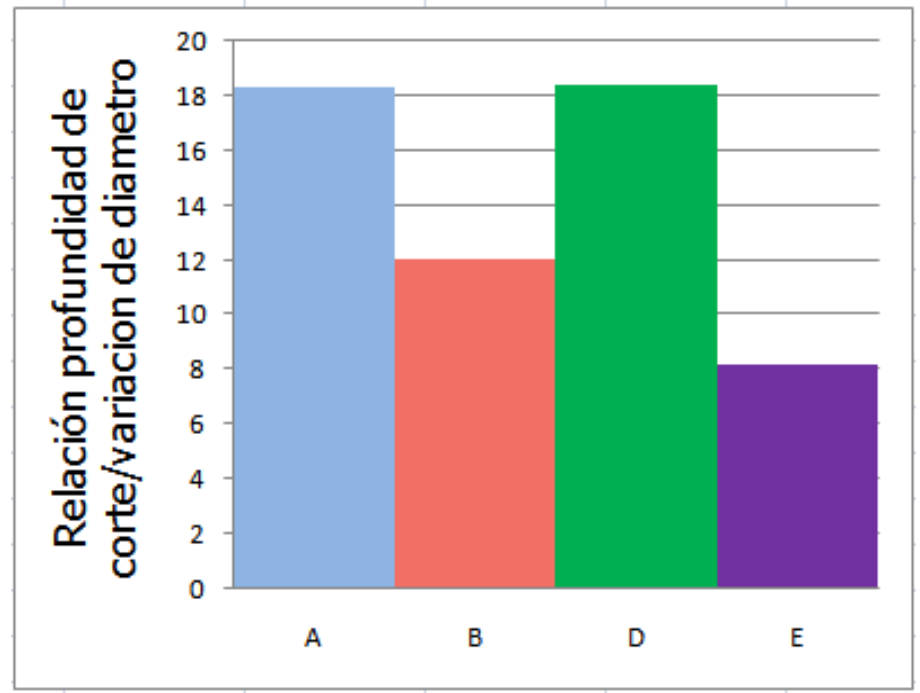

Figura 7: Profundidad de corte / variación diametral.

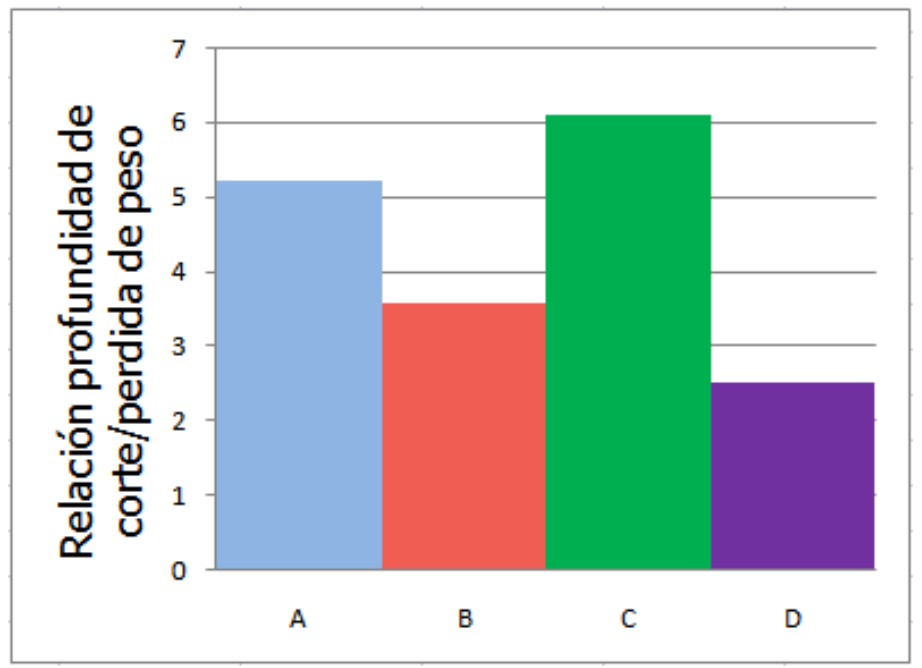

Figura 8: Profundidad de corte / pérdida de peso.

El análisis conjunto de estos dos diagramas muestra que el mejor comportamiento global lo tuvo el grupo de discos C. Sin embargo, no hay que dejar de lado que, el grupo A fue aquel que presentó una elevada capacidad de corte en términos de la profundidad, aunque con un desgaste algo mayor que el grupo C.

Por último, analizamos el motivo por el cual algunas de las marcas cortaron unos $20 \mathrm{~mm}$ de profun- 
didad h durante todo el ensayo (marcas A y B), en tanto que otra sólo $8 \mathrm{~mm}$ (marca D). Para ello, se extrajeron un segmento desgastado del disco B (verde - representativo de un disco con alta profundidad de corte) y otro del disco D (violeta - baja profundidad de corte).

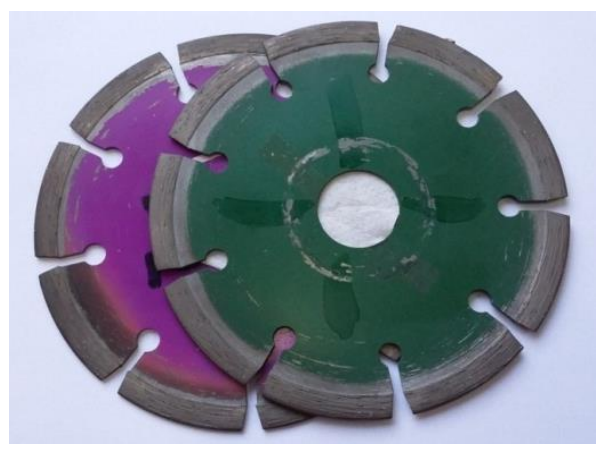

Figura 9: Imagen del disco D (izquierda) y disco B.

Estos segmentos fueron examinados por microscopía electrónica, tomándose imágenes sobre las superficies laterales, a la vez que se midieron las alturas máximas de los diamantes respecto del aglutinante.

Seguidamente se muestra una imagen representativa de cada una de las muestras analizadas.
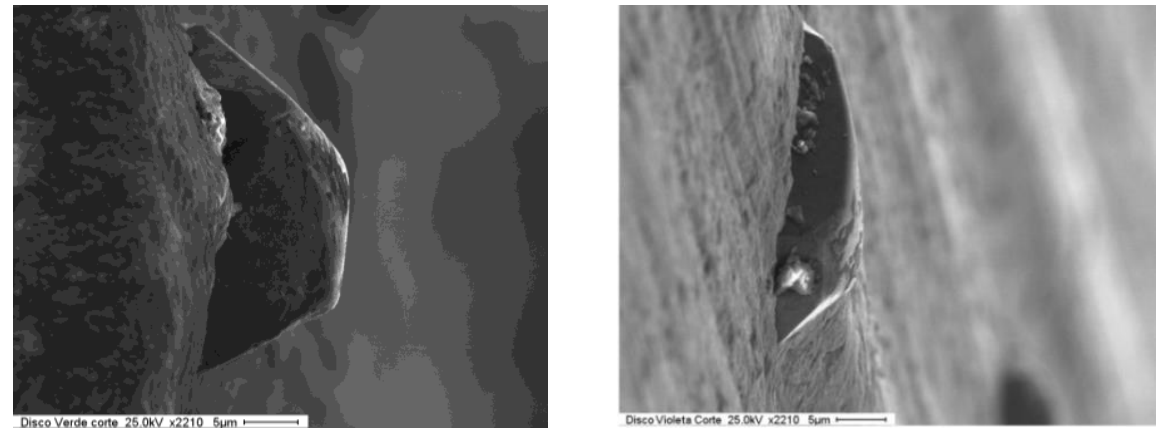

Figura 10: Grano de diamante disco B (izquierda) y de disco D.

Un aspecto particular fue que, en el sector analizado del disco D, se observaron huecos ocasionados por desprendimiento de granos de diamante, situación que no ocurrió en el disco de corte B (está claro que esta situación afecta la capacidad cortante).

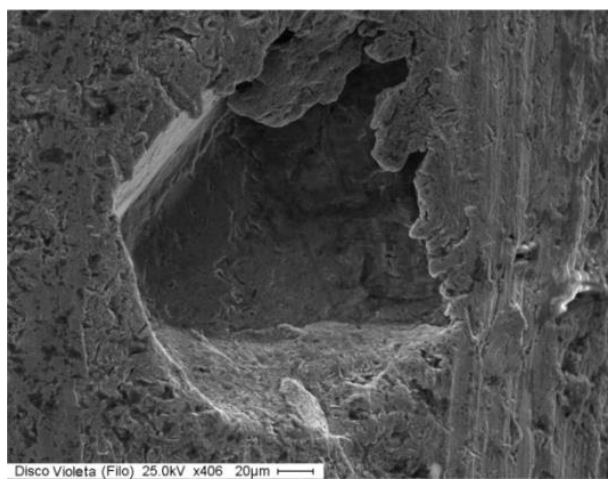

Figura 11: Hueco provocado por desprendimiento de diamante en muestra de disco D.

A partir de las imágenes obtenidas resultó evidente una diferencia en la altura máxima de cada uno de 
los granos de diamante que sobresalen respecto del aglutinante (ver figura 10), si bien el conteo de granos fue similar. Por lo tanto, se procedió a cuantificar las alturas sobresalientes midiendo 10 granos en cada muestra.

Los resultados se presentan en la tabla siguiente:

Tabla 4: Altura máxima de los granos respecto del aglutinante

\begin{tabular}{l|l|l}
\hline DISCO & ALTURA MÁXIMA PROMEDIO $(\boldsymbol{\mu m})$ & DESVIACIÓN ESTÁNDAR $(\%)$ \\
\hline Marca B (verde) & 10,4 & $\pm 11,8$ \\
\hline Marca D (violeta) & 6,2 & $\pm 10,7$ \\
\hline
\end{tabular}

En vista de los resultados obtenidos, imágenes y valores medidos, se puede inferir que, los diamantes del disco B, que mantuvieron su capacidad de corte h en unos $20 \mathrm{~mm}$, sobresalieron en promedio un $68 \%$ más que aquellos diamantes del disco $\mathrm{D}$, y consecuentemente una mayor altura es sinónimo de poder realizar cortes más profundos.

\section{CONCLUSIONES}

- En este trabajo, se presenta un procedimiento completo sobre como evaluar la performance de discos segmentados de diamante. Para tal fin fue necesario desarrollar: una máquina de corte $\mathrm{CNC}$, un material de ensayo, las condiciones operativas, el criterio de interrupción, y la forma de evaluación (desgaste y profundidad de corte).

- Los discos de los grupos A y D mostraron un comportamiento similar frente al desgaste, aunque llamativamente los del grupo D, sólo pudieron cortar profundidades de unos $8 \mathrm{~mm}$.

- En cuanto al desgaste, el grupo B resultó el de menor performance, mientras que el C alcanzó la mejor.

- En relación a la capacidad de cortar mayores profundidades $(h)$, los discos de los grupos A y B fueron aquellos de mejor comportamiento.

- Si el comportamiento del disco se juzga por la relación profundidad de corte / desgaste, el mejor comportamiento global lo tuvo el grupo de discos C.

- De nada sirve disponer de un disco que se desgaste poco, sino permite realizar cortes profundos. Tal es el caso del grupo D.

- De acuerdo con los resultados obtenidos, es posible afirmar que, los métodos de variación del diámetro y de pérdida de peso, usados para cuantificar el desgaste de los discos, pueden considerarse equivalentes.

- La observación de las superficies de corte de este tipo de discos, empleando microscopía electrónica de barrido, permite justificar la capacidad cortante en términos de la profundidad de corte, evidenciada durante los ensayos.

- Finalmente, el trabajo aporta un criterio de interrupción basado en la superficie lateral cortada, la posibilidad de evaluar el desgaste por la variación diametral de los discos con el uso, la existencia de una relación en la capacidad de corte en términos de la profundidad con la altura que los granos de diamante sobresalen del aglutinante, y un criterio de evaluación basado en la relación profundidad de corte / desgaste.

\section{AGRADECIMIENTOS}

A nuestros compañeros del Laboratorio de Microscopía Electrónica de INTI-Mecánica. 


\section{BIBLIOGRAFÍA}

[1] CRUZ FERNANDEZ, J., "Development of a new testing method for assesing the wear behaviour of a circular cuttings discs", Materials Science Forum, v. 587-588, pp. 966-970, Jun. 2008.

[2] GUERRA ROSA, L. "Test Methodology to evaluate the wear performance of PM matrices used in Diamond impregnated tools for cutting hard materials", In: P. Ramakrishnan (ed), Power Metallurgy for Automotive, 1 ed., chapter 22, New Delhi, India, New Age International Ltd, 2012.

[3] UCUN, I., "Effect of cooling liquids on cutting process using diamond segmented disc of natural stones", Proceedings of the Institution of Mechanical Engineers Part C: Journal of Mechanical Engineering Science Journal of Mechanical Engineering Science, v. 203-210, Jul. 2013.

[4] UCUN, I., "An investigation on the effect of diamond concentration and matrix material composition in the circular sawing process of granites", Proceedings of the Institution of Mechanical Engineers Part C: Journal of Mechanical Engineering Science Journal of Mechanical Engineering Science, v. 203-210, Jan. 2010.

[5] ASLANTAS, K., "Investigation of the effect of axial cutting force on circular Diamond saw blade used in marble cutting process", Materials and Manufacturing Processes, v. 24, pp. 1423-1430, Dec. 2009.

[6] ANJINHO, C., "New standard methodologies of classify the efficiency of Diamond cutting discs", Global Stone Congress 2010, Alicante, Spain, March 2010.

[7] UCUN, I., "Determination of specific energy in cutting process using diamond saw blade of natural stone", Energy Education Science and Technology Part A: Energy Science and Research, v. 28, pp. 641-648, 2012. 